\title{
Where are the strongest associations between autistic traits and traits of ADHD? evidence from a community-based twin study
}

\author{
Mark J. Taylor · Tony Charman • Angelica Ronald
}

Received: 16 May 2014 / Accepted: 13 December 2014 / Published online: 20 January 2015

(C) Springer-Verlag Berlin Heidelberg 2015

\begin{abstract}
Autism spectrum conditions (ASC) and attention-deficit/hyperactivity disorder (ADHD) regularly cooccur. Twin studies increasingly indicate that these conditions may have overlapping genetic causes. Less is known about the degree to which specific autistic traits relate to specific behaviours characteristic of ADHD. We hence tested, using the classical twin design, whether specific dimensional autistic traits, including social difficulties, communication atypicalities and repetitive behaviours, would display differential degrees of aetiological overlap with specific traits of ADHD, including hyperactivity/ impulsivity and inattention. Parents of approximately 4,000 pairs of 12-year-old twins completed the Childhood Autism Spectrum Test and Conners' Parent Rating Scale. These measures were divided into subscales corresponding to different types of autistic and ADHD behaviours. Twin model fitting suggested that the degree of genetic overlap was particularly strong between communication difficulties and traits of ADHD (genetic correlations $=.47-.51$ ), while repetitive behaviours and social difficulties showed moderate (genetic correlations $=.12-.33$ ) and modest $(.05-.11)$ genetic overlap respectively. Environmental overlap was low across all subscales (correlations $=.01-.23$ ). These
\end{abstract}

Electronic supplementary material The online version of this article (doi:10.1007/s00787-014-0666-0) contains supplementary material, which is available to authorized users.

M. J. Taylor $(\bowtie) \cdot$ A. Ronald

Genes Environment Lifespan Laboratory, Centre for Brain and Cognitive Development, Department of Psychological Science, Birkbeck, University of London, London, UK e-mail: mj.taylor@bbk.ac.uk

T. Charman

Department of Psychology, King's College London, London, UK patterns were also apparent at the extremes of the general population, with communication difficulties showing the highest genetic overlap with traits of ADHD. These findings indicate that molecular genetic studies seeking to uncover the shared genetic basis of ASC and ADHD would benefit from taking a symptom-specific approach. Furthermore, they could also help to explain why studies of the communication abilities of individuals with ASC and ADHD have produced overlapping findings.

Keywords Autism · ADHD · Twin study - Genetics . Comorbidity

\section{Introduction}

Based on their diagnostic criteria, autism spectrum conditions (ASC) and attention-deficit/hyperactivity disorder (ADHD) have little in common. ASC are characterised by atypical social interaction, communication difficulties, and behavioural inflexibility, while ADHD is defined by excessive hyperactivity, impulsivity, and/or inattentiveness [1]. Yet it is common for these conditions to co-occur; ADHD is thought to present in 30-60\% of individuals with ASC $[2,3]$, while autistic traits appear elevated in individuals with ADHD [4]. It is thus necessary for research to explore why these conditions so often co-occur with one another.

ASC and ADHD are highly heritable [5, 6]. One possible explanation for their co-occurrence could be that their genetic and environmental causes overlap with one another. This assertion has been supported by a multitude of recent twin studies. These studies have reported a moderate degree of genetic overlap between ASC and ADHD, both as dimensional characteristic traits and diagnosed conditions, in middle childhood [7], early adolescence [8-10], 
early adulthood [11], and adulthood [10]. These studies also report a modest $[7-9,11]$ to moderate $[10]$ degree of environmental overlap across these traits.

There is converging evidence from family and twin studies that the core autistic traits, social and communication difficulties and restricted repetitive behaviours, have largely different causes [12-16]; that is, certain genes or environments may be associated with social difficulties, for example, but may not cause communication difficulties or rigid behavioural patterns. There is also evidence of some genetic specificity across traits of ADHD [17-19]. As such, it is possible that the degree of aetiological overlap between autistic traits and ADHD behaviours differs across particular types of these traits. Two recent twin studies tested this possibility. A Dutch study reported that attention switching difficulties, rather than social autistic traits, showed particularly strong genetic overlap with traits of ADHD [20]. A study of a Swedish cohort found that repetitive routines and behaviours showed elevated genetic overlap with traits of ADHD [21]. Together, these studies indicate that traits of ADHD are particularly associated with non-social autistic traits.

There have yet to be any twin studies of these associations in extreme-scoring groups. Individuals displaying extreme scores on trait measures typically display scores that are more comparable to clinical groups, thus indicating whether or not we would expect trait findings to extend to clinical groups. For this reason, we conducted a twin study aimed at testing the degree of genetic overlap between specific autistic traits, including social difficulties, communication atypicalities, and repetitive routines and behaviours, and specific traits of ADHD, including hyperactivity/ impulsivity and inattention, at the extremes of the general population. We also further tested genetic and environmental overlap between these traits across the general population. We hypothesised that the degree of aetiological overlap between autistic traits and traits of ADHD would differ according to different types of autistic and ADHD trait. We further expected repetitive routines and behaviours to show stronger aetiological overlap with traits of ADHD than social and communicative autistic traits.

\section{Method}

Participants

Parents of 5,879 twins participating in the Twins Early Development Study (TEDS) completed and returned questionnaires when twins were aged 12. TEDS is a community-based, population representative study of twins born in England and Wales between 1994 and 1996 [22]. Exclusions were conducted for genetic syndromes, including Fragile X and cystic fibrosis, chromosomal abnormalities, including
Down Syndrome and cerebral palsy, extreme perinatal or prenatal difficulties, and missing first contact or zygosity data. Participants with confirmed ASC $(N=80)$ were included. The final sample comprised 5,689 twin pairs, including 927 monozygotic (MZ) male pairs, 1,124 MZ female pairs, 865 dizygotic (DZ) male pairs, 989 DZ female pairs, and 1,784 DZ opposite-sex pairs. Zygosity was ascertained through DNA testing and parent ratings of twin resemblance [23]. All participants provided written informed consent prior to participation. TEDS has full ethical approval from the King's College London Research Ethics Committee.

\section{Measures}

\section{Autistic traits}

Parents completed the Childhood Autism Spectrum Test (CAST [24]), a 30-item questionnaire that enquires about various autistic traits, such as lack of social interest, pronounced interests, and difficulties with conversation. Each question was answered 'yes' or 'no', meaning the maximum possible score was 30. Scores over 15 predict ASC diagnoses with $100 \%$ sensitivity and $97 \%$ specificity [25]. The measure was divided into three scales based on DSM-IV criteria, which were employed in previous studies [13, 14]: social difficulties ('Social'), communication atypicalities ('Communication'), and repetitive, restricted behaviours and interests (RRBI). The number of items, possible range of scores, and Cronbach's $\alpha$ values for each subscale are shown in Table 1.

\section{Traits of $A D H D$}

Parents completed the ADHD subscale of the Conners' Parent Rating Scale ('Conners ADHD' [26] ), a measure formed of 18 statements. Each statement described a behaviour characteristic of ADHD; parents rated, on a three-point scale, how true each statement was of their child. The highest possible score was 54. Conners ADHD is a valid assessment of ADHD traits; individuals with ADHD display significantly higher scores on the measure than controls [26]. Consistent with previous recommendations [26], the measure was divided into two subscales, which closely corresponded to DSM-IV criteria for ADHD: Hyperactivity/Impulsivity and Inattention. Possible ranges of scores, number of items, and Cronbach's $\alpha$ for both subscales are provided in Table 1.

Data analysis

\section{Twin analyses of the full sample}

Data were prepared for analysis by first log transforming any skewed subscales (see Table 1), and then regressing the 
Table 1 Descriptive statistics, phenotypic correlations, and twin correlations

\begin{tabular}{|c|c|c|c|c|c|c|}
\hline Measure & $\begin{array}{l}\text { Number } \\
\text { of items }\end{array}$ & Cronbach's $\alpha$ & Skewness & $\begin{array}{l}\bar{x} \text { Full sample } \\
(95 \% \text { CI })\end{array}$ & $\begin{array}{l}\bar{x} 5 \% \text { extremes } \\
(95 \% \mathrm{CI})\end{array}$ & Probands $(N)$ \\
\hline CAST social & 11 & 0.54 & $1.42(-0.01)$ & $1.61(1.58 / 1.64)$ & $5.91(5.80 / 6.01)$ & 546 \\
\hline CAST communication & 12 & 0.64 & $1.36(0.02)$ & $1.90(1.87 / 1.93)$ & $7.01(6.91 / 7.12)$ & 590 \\
\hline CAST RRBI & 7 & 0.49 & $.96(-0.05)$ & $1.41(1.39 / 1.44)$ & $4.47(4.42 / 4.57)$ & 747 \\
\hline Conners ADHD hyperactivity/impulsivity & 9 & 0.84 & $1.70(-0.09)$ & $4.30(4.22 / 4.38)$ & $16.85(16.57 / 17.14)$ & 656 \\
\hline Conners ADHD inattention & 9 & 0.90 & $1.38(-0.32)$ & $5.58(5.48 / 5.67)$ & $19.89(19.63 / 20.14)$ & 632 \\
\hline
\end{tabular}

Skewness values are first given for untransformed scales; the values in parentheses are for transformed, standardised residuals used in the analyses CAST Childhood Autism Spectrum Test, CAST social CAST social difficulties subscale, CAST communication CAST communication atypicalities subscale; CAST RRBI CAST repetitive, restricted behaviours and interests subscale

effects of sex and age out of all measures, as is standard behavioural genetic procedure [27]. Analyses were then performed on standardised residual scores using Mx [28].

First, phenotypic associations between the CAST and Conners ADHD subscales were established using phenotypic correlations $\left(r_{\mathrm{ph}}\right)$, correlation coefficients estimated from structural equation models. Second, twin correlations, which are the foundation of twin analysis, were estimated. Cross-trait cross-twin correlations provide one with an initial indication of the extent to which covariance between two traits is influenced by genetic and environmental factors. These involved correlating one twin's score on a CAST subscale with their co-twin's score on a Conners ADHD subscale. Cross-trait cross-twin correlations were estimated separately for MZ and DZ twins. These estimates cannot exceed $r_{\mathrm{ph}}$ between two traits. As MZ twins are assumed to share all of their segregating DNA code with one another, while DZ twins share, on average, $50 \%$, additive genetic (A) influences on the covariance between two traits are implicated if the MZ cross-trait cross-twin correlation exceeds the DZ estimate. As MZ twins are assumed to be genetically identical, any within pair MZ differences are assumed to be caused by nonshared environment (E), environmental factors that differ across twins in a pair and create differences between them (measurement error is included in this term). Hence, if the MZ cross-trait crosstwin correlation between two traits is less than $r_{\mathrm{ph}}$ between them, $\mathrm{E}$ is implicated on their covariance. Shared environmental $(\mathrm{C})$ influences, on the other hand, are common to both twins in a pair and heighten their similarity; these influences are indicated if the DZ cross-trait cross-twin correlation exceeds half the MZ statistic. Alternatively, nonadditive genetic (D) influences are implicated if the DZ cross-trait cross-twin correlation is less than half the MZ estimate. These correlations were estimated from a constrained saturated model (see below).

Finally, multivariate structural equation twin models were fitted to data to formally estimate A, C, D, and E. Cholesky decompositions, presented here as correlated factors solutions [29], were tested. For each trait, A, C (or $\mathrm{D}$, if this is suggested by the twin correlations), and $\mathrm{E}$ were estimated. The additive genetic correlation $\left(r_{\mathrm{A}}\right)$ then estimates A overlap between traits. This estimate falls between 0 and 1; estimates of 1 suggest total genetic overlap across two traits, while estimates of 0 would suggest that the genetic influences on two traits are totally independent of one another. Shared environmental $\left(r_{\mathrm{C}}\right)$, nonshared environmental $\left(r_{\mathrm{E}}\right)$, and non-additive genetic $\left(r_{\mathrm{D}}\right)$ correlations can also be computed, and operate in the same manner. The model then computes bivariate heritability, which estimates the extent to which A can explain $r_{\text {ph }}$ between two traits, from the univariate $\mathrm{A}$ estimates and $r_{\mathrm{A}}$. Equivalent statistics are calculated for $\mathrm{C}, \mathrm{E}$, and $\mathrm{D}$.

ACE and ADE models were tested. Parameters in these models can either be equated across sexes, or allowed to differ in magnitude across sexes (quantitative sex differences). Such differences are indicated when male and female twin correlations differ. In order to test for quantitative sex differences, a saturated model that allowed twin correlations to differ by sex was fitted. These estimates were then equated across sexes.

The fit of each model was compared against that of a saturated model of the observed data. For each model, the $-2 \mathrm{LL}$ fit statistic was computed. Model fit was assessed using the likelihood-ratio test (LRT), which capitalises on the fact that differences in $-2 \mathrm{LL}$ between two models are $\chi^{2}$ distributed, with degrees of freedom equal to the difference in number of estimated parameters. Significant $\chi^{2}$ results suggest that a given model is a significantly poorer fit than the saturated model. Model fit was further assessed using Akaike's Information Criteria (AIC) and Bayesian Information Criteria (BIC). Lower, preferably negative, AIC values indicate good model fit, while models with more negative BIC estimates are to be favoured. Selection of the best-fitting model was based on BIC, which performs better than either the LRT or AIC in larger samples [30]; when comparing two models, the model with the more negative BIC estimate is regarded as better-fitting, 
with differences of 10 or more argued to show that a given model is a good fit [31].

\section{Analysis of the extremes}

DeFries-Fulker analysis [32, 33] was used to analyse extreme scores on the CAST and Conners ADHD subscales. DeFries-Fulker analysis is a regression analysis of means. The univariate procedure is designed to estimate group heritability $\left(h_{\mathrm{g}}^{2}\right)$, which refers to genetic influences on extreme scores [30]. Bivariate DeFries-Fulker analysis is concerned with testing for bivariate heritability $\left(h_{2 . x y}\right)$; in these analyses, this refers to the extent to which the genes that cause extreme scores on one trait influence continuous scores on another trait [32]. In performing univariate and bivariate DeFries-Fulker analyses, it is possible to estimate genetic correlations between extreme scores on two measures [33].

For the univariate DeFries-Fulker analyses, extreme scorers, termed probands, were selected from the sample used in the twin analyses on the basis of scoring within the highest $5 \%$ of the z-score distribution on a measure of interest. Scores were then transformed so that the mean proband score on the measure of interest was 1 , while the control mean was 0 . In transforming scores in this manner, one can gain an indication of $h_{\mathrm{g}}^{2}$ by examining the mean scores of co-twins of probands. If the DZ co-twin mean is closer to 0 than the MZ co-twin mean, then greater resemblance between MZ twins than DZ twins is suggested. Much like how one interprets twin correlations, this can be taken as evidence of $h_{\mathrm{g}}^{2}$ on a trait. Univariate DeFries-Fulker analysis more formally estimates $h_{\mathrm{g}}^{2}$ through a regression equation for predicting co-twin scores on a given measure from proband scores and zygosity. The regression coefficient of co-twin scores on zygosity is an estimate of $h_{\mathrm{g}}^{2}$. The estimate of $h_{\mathrm{g}}^{2}$ should not exceed the mean scores of $\mathrm{MZ}$ co-twins, unless non-additive genetic effects are influential. In this instance, estimates were fixed to equal the mean MZ co-twin score.

In bivariate DeFries-Fulker analysis, probands were selected on the basis of scoring within the highest $5 \%$ of the z-score distribution on a given measure; the measure used to select probands is the selection variable. The outcome variable is the second measure of interest. Thus, the bivariate procedure is directional in that a CAST subscale could be used as the selection variable and a Conners ADHD subscale used as the outcome variable, and vice versa.

Prior to conducting bivariate DeFries-Fulker analysis, the phenotypic associations between the selection and outcome variables were tested using phenotypic group correlations. These were calculated by dividing proband's mean $\mathrm{z}$-score on the selection variable by their mean $\mathrm{z}$-score on the outcome variable, and can be interpreted in the same manner as correlation coefficients. Subsequently, data were prepared for bivariate analysis by dividing scores on both measures by the zygosity-specific proband mean for the selection variable [34]. Transforming scores in this manner meant that the mean score on the selection variable for probands was 1 , while the mean score for controls on the outcome measure was 0 . The co-twin means provide an indication of the extent of $h_{2 . x y}$ between two measures; if the DZ co-twin mean on the outcome variable is closer to 0 than $\mathrm{MZ}$ co-twin means, $h_{2 . \mathrm{xy}}$ between the selection and outcome variables is indicated. The bivariate DeFriesFulker regression equation, which predicts co-twin scores on the outcome measure from proband scores on the selection measure and zygosity, was then used to formally estimate $h_{2 . x y}$. The regression coefficient of co-twin scores on zygosity is an estimate of $h_{2 . x y}$. Again, this estimate only exceeds the mean scores on the outcome variable for MZ co-twins if non-additive genetic influences are in operation; as in the univariate analysis, $\mathrm{h}_{2 . x y}$ was fixed to equal the $\mathrm{MZ}$ co-twin mean for the outcome variable in these instances. These analyses were conducted using the CAST subscales as selection variables, and then using the Conners ADHD subscales as selection variables.

The results of the univariate and bivariate DeFriesFulker analyses were then used to calculate genetic correlations between extreme CAST subscale scores and extreme Conners ADHD subscale scores, using the equation: $\sqrt{\frac{\left(\beta_{x y} \times \beta_{y x}\right)}{\left(\beta_{x} \times \beta_{y}\right)}}$ [35]. $\beta_{x y}$ and $\beta_{y x}$ represent $h_{2 . x y}$ estimates, while $\beta_{x}$ and $\beta_{y}$ are $h_{\mathrm{g}}^{2}$ estimates for both measures.

\section{Results}

Full sample

Descriptive statistics for all measure subscales are shown in Table 1. Table 2 shows $r_{\text {ph }}$ estimates. The highest estimate was between Communication and Hyperactivity/Impulsivity (.43), followed by Communication and Inattention (.40). RRBI displayed modest $r_{\text {ph }}$ with Hyperactivity/Impulsivity (.26) and Inattention (.05), while associations were weakest between Social and hyperactivity/impulsivity (.06) and Inattention (.07).

Twin correlations are shown in Table 3. These suggest that each CAST and Conners ADHD subscale was heritable. Cross-trait cross-twin correlations suggested that the covariance between the CAST and Conners ADHD subscales was largely influenced by genetic factors; with the exception of the cross-trait cross-twin correlations between RRBI and Inattention, MZ estimates exceeded DZ estimates. Many of the DZ estimates were also less than half 
Table 2 Phenotypic correlations

\begin{tabular}{llc}
\hline $\begin{array}{l}\text { Conners ADHD } \\
\text { CAST subscale }\end{array}$ & Conners ADHD hyperactivity/impulsivity & Conners ADHD inattention \\
subscale & & \\
\hline CAST social & $0.06^{*}$ & $0.07^{*}$ \\
CAST communication & $0.43^{*}$ & $0.40^{*}$ \\
CAST RRBI & $0.26^{*}$ & $0.05^{*}$ \\
\hline
\end{tabular}

CAST Childhood Autism Spectrum Test, CAST social CAST social difficulties subscale, CAST communication CAST communication atypicalities subscale; CAST RRBI CAST repetitive, restricted behaviours and interests subscale, Conners ADHD Conners' Parent Rating Scale, Conners ADHD hyperactivity/impulsivity Conners hyperactivity/impulsivity subscale, Conners Inattention Conners inattention subscale

$* p<0.05$

Table 3 Twin correlations

\begin{tabular}{|c|c|c|c|c|}
\hline \multicolumn{5}{|l|}{ Cross-twin correlations } \\
\hline \multirow[t]{2}{*}{ Measure } & \multicolumn{2}{|c|}{ Monozygotic twins } & \multicolumn{2}{|c|}{ Dizygotic twins } \\
\hline & Estimate & $95 \% \mathrm{CI}$ & Estimate & $95 \% \mathrm{CI}$ \\
\hline CAST Social & 0.72 & $0.70-0.74$ & 0.34 & $0.30-0.38$ \\
\hline CAST Communication & 0.76 & $0.74-0.77$ & 0.39 & $0.35-0.43$ \\
\hline CAST RRBI & 0.71 & $0.69-0.73$ & 0.30 & $0.26-0.34$ \\
\hline Conners Hyp/Imp & 0.88 & $0.87-0.89$ & 0.50 & $0.46-0.53$ \\
\hline Conners Inattention & 0.78 & $0.76-0.79$ & 0.37 & $0.33-0.41$ \\
\hline \multicolumn{5}{|l|}{ Cross-trait cross-twin correlations } \\
\hline \multirow[t]{2}{*}{ Measures } & \multicolumn{2}{|c|}{ Monozygotic twins } & \multicolumn{2}{|c|}{ Dizygotic twins } \\
\hline & Estimate & $95 \% \mathrm{CI}$ & Measure & Estimate \\
\hline CAST Social-Conners Hyp/Imp & 0.12 & $0.07-0.16$ & 0.08 & $0.07-0.16$ \\
\hline CAST Social-Conners Inatt. & 0.13 & $0.09-0.17$ & 0.08 & $0.04-0.13$ \\
\hline CAST comm.-Conners Hyp/Imp & 0.44 & $0.41-0.48$ & 0.27 & $0.23-0.31$ \\
\hline CAST comm.-Conners Inatt. & 0.41 & $0.37-0.45$ & 0.19 & $0.14-0.23$ \\
\hline CAST RRBI-Conners Hyp/Imp & 0.26 & $0.22-0.30$ & 0.21 & $0.17-0.26$ \\
\hline CAST RRBI-Conners Inatt. & 0.10 & $0.06-0.15$ & 0.11 & $0.06-0.15$ \\
\hline
\end{tabular}

CAST Childhood Autism Spectrum Tes, CAST social CAST social difficulties subscale, CAST communication/CAST comm. CAST communication atypicalities subscale, CAST RRBI CAST repetitive, restricted behaviours and interests subscale, Conners hyp/imp Conners' Parent Rating Scale (Conners ADHD) hyperactivity/impulsivity subscale, Conners inattention/Conners inatt. Conners ADHD inattention subscale

the MZ estimates, indicating that $\mathrm{D}$ may have played a role in the covariance between some of the subscales. No MZ cross-trait cross-twin correction exceeded $\mathrm{r}_{\mathrm{ph}}$, indicating $\mathrm{E}$.

Fit statistics for the twin models tested are given in Table 4. A saturated model that equated twin correlations across sexes fitted better than one that allowed them to differ by sex, as indicated by a considerably lower BIC estimate for the constrained saturated model $(-157,379.80)$ than for the saturated model with sex differences $(-156,485.70)$. ACE and ADE models were thus tested with equivalent estimates in both sexes. Models included all three CAST and both Conners ADHD subscales. Relative to the saturated model, an ADE correlated factors solution had most negative BIC value $(-157,928.84)$. In dropping
$\mathrm{D}$ from the model, BIC $(-158,004.70)$ reduced further, hence an AE model was chosen as the best fitting model. Parameter estimates from the AE model are all shown in Table 5. All three CAST subscales were heritable, with A explaining $69-72 \%$ of the variance in each. The same was true for both Conners ADHD subscales $(\mathrm{A}=75-86 \%)$. The aetiological correlations between the measure subscales suggested that the strongest degree of genetic overlap was between Communication and both Hyperactivity/ Impulsivity $\left(r_{\mathrm{A}}=.50\right)$ and Inattention $\left(r_{\mathrm{A}}=.48\right)$. Genetic correlations between the CAST communication difficulties subscale and traits of ADHD were significantly stronger than all other genetic correlations; there was moderate A overlap between RRBI and Hyperactivity/Impulsivity 
Table 4 Twin model fit statistics

\begin{tabular}{|c|c|c|c|c|c|c|c|c|}
\hline Model & $-2 \mathrm{LL}$ & $d f$ & Parameters & $\mathrm{BIC}$ & $\Delta \chi^{2}$ & $\Delta d f$ & $p$ & AIC \\
\hline Saturated & $66,869.83$ & 27,116 & 130 & $-157,379.84$ & - & - & - & - \\
\hline ACE & $67,001.24$ & 27,196 & 50 & $-157,910.04$ & 131.41 & 80 & $<0.001$ & -28.59 \\
\hline $\mathrm{ADE}$ & $66,982.44$ & 27,196 & 50 & $-157,928.84$ & 112.61 & 80 & $<0.05$ & -47.39 \\
\hline
\end{tabular}

Submodels

Model $-2 \mathrm{LL} \quad d f \quad$ Parameters BIC

Comparative fit with saturated model Comparative fit with ADE model

\begin{tabular}{|c|c|c|c|c|c|c|c|c|c|c|c|c|}
\hline & & & & & & & & & & & & \\
\hline & & & & & $\Delta \chi^{2}$ & $\Delta d f$ & $p$ & AIC & $\Delta \chi^{2}$ & $\Delta d f$ & $p$ & AIC \\
\hline $\mathrm{AE}^{\mathrm{a}}$ & $67,030.60$ & 27,211 & 35 & $-158,004.70$ & 160.77 & 95 & $<0.001$ & -29.23 & 48.17 & 15 & $<0.001$ & 18.17 \\
\hline $\mathrm{E}$ & $73,177.18$ & 27,226 & 20 & $-151,982.20$ & $6,307.35$ & 110 & $<0.001$ & $6,087.35$ & $6,194.74$ & 30 & $<0.001$ & $6,134.74$ \\
\hline
\end{tabular}

All submodels are nested within the ADE model, which was a better fit relative to the saturated model than the ACE model

$-2 L L$ fit statistic, BIC Bayesian Information Criteria, AIC Akaike's Information Criteria, $\Delta \chi^{2}$ change in -2LL between two models, Chi square distributed

a Indicates best fitting model; $p$ values are corrected for multiple comparisons using the Bonferoni correction

Table 5 Estimates derived from the best fitting correlated factors solution

\begin{tabular}{|c|c|c|c|c|}
\hline & \multicolumn{2}{|l|}{ A } & \multicolumn{2}{|l|}{$\mathrm{E}$} \\
\hline & Estimate & $95 \% \mathrm{CI}$ & Estimate & $95 \% \mathrm{CI}$ \\
\hline \multicolumn{5}{|c|}{ Variance Components Estimates } \\
\hline Social & 0.72 & $0.68-0.75$ & 0.28 & $0.27-0.30$ \\
\hline Comm. & 0.71 & $0.69-0.73$ & 0.29 & $0.27-0.30$ \\
\hline RRBI & 0.69 & $0.66-0.70$ & 0.31 & $0.30-0.33$ \\
\hline $\mathrm{H} / \mathrm{I}$ & 0.86 & $0.84-0.87$ & 0.14 & $0.12-0.15$ \\
\hline \multirow[t]{2}{*}{ Inatt. } & 0.75 & $0.74-0.76$ & 0.25 & $0.22-0.26$ \\
\hline & \multicolumn{2}{|c|}{$r_{\mathrm{A}}$} & \multicolumn{2}{|l|}{$r_{\mathrm{E}}$} \\
\hline \multicolumn{5}{|c|}{ Aetiological Correlations } \\
\hline Social-H/I & & -0.01 to 0.09 & -0.02 & -0.09 to 0.05 \\
\hline Socia-Inatt. & & 0.01 to 0.10 & 0.03 & -0.01 to 0.06 \\
\hline Comm.-H/I & & 0.44 to 0.56 & 0.22 & 0.21 to 0.24 \\
\hline Comm.-Inatt. & & 0.41 to 0.49 & 0.19 & 0.17 to 0.20 \\
\hline $\mathrm{RRBI}-\mathrm{H} / \mathrm{I}$ & & 0.22 to 0.34 & 0.11 & 0.01 to 0.12 \\
\hline RRBI-Inatt. & & 0.09 to 0.18 & -0.03 & -0.05 to 0.01 \\
\hline
\end{tabular}

Bivariate heritability and nonshared environment

\begin{tabular}{llll} 
Conners ADHD hyperactivity/impulsivity & & \multicolumn{2}{l}{ Conners ADHD Inattention } \\
\cline { 4 - 5 } Bivariate heritability \% & Bivariate nonshared environment $\%$ & & Bivariate heritability \%
\end{tabular}

Bivariate nonshared environment $\%$

\begin{tabular}{|c|c|c|c|c|}
\hline CAST social & 100 & 0 & 86 & 14 \\
\hline CAST communication & 91 & 9 & 88 & 12 \\
\hline CAST RRBI & 92 & 8 & 80 & 20 \\
\hline
\end{tabular}

All italicised estimates were not significant, as indicated by confidence intervals overlapping with zero

CAST Childhood Autism Spectrum Test, Social social difficulties subscale of the CAST, Communication/Comm. communication atypicalities subscale of the CAST, RRBI repetitive, restricted behaviours and interests subscale of the CAST, Conners ADHD Conners' Parent Rating Scale, Hyp/imp hyperactivity/impulsivity subscale of conners adhd,Inatt inattention subscale of Conners ADHD, $A$ additive genetic influences, $E$ nonshared environmental influences, $r_{A}$ additive genetic correlation, $r_{E}$ nonshared environmental correlation 
$\left(r_{\mathrm{A}}=.31\right)$. However, $\mathrm{r}_{\mathrm{A}}$ was low between all other subscales $\left(r_{\mathrm{A}}=.08-.14\right)$. Nonshared environmental overlap between the three CAST subscales and Conners ADHD subscales was low. The highest $r_{\mathrm{E}}$ estimates were between Communication and Hyperactivity/Impulsivity $\left(r_{\mathrm{E}}=22\right)$ and Inattention $\left(r_{\mathrm{E}}=.19\right)$. This was again significantly higher than the estimates seen between other subscales. RRBI displayed greater E overlap with hyperactivity/ impulsivity $\left(r_{\mathrm{E}}=.11\right)$ than Inattention $\left(r_{\mathrm{E}}=-.03\right)$, while $\mathrm{r}_{\mathrm{E}}$ between Social and Conners ADHD subscales was low and largely non-significant.

The majority of $r_{p h}$ between the CAST and Conners ADHD subscales could be explained by A (see Table 5). Bivariate heritability was estimated as at least $80 \%$ got each $r_{\mathrm{ph}}$ estimate, with estimates of bivariate heritability peaking at $100 \%$. E, by contrast, explained no greater than $20 \%$ of each $r_{\mathrm{ph}}$ estimate. These results did not significantly change when individuals with ASC were removed from the analyses (contact the first author for further details).

\section{Extremes analysis}

Results of the DeFries-Fulker analysis are provided in full in the supplementary materials. Descriptive statistics for the CAST and Conners ADHD subscales in the $5 \%$ extreme groups, along with the number of probands in each group, are given in Table 1.

Table S1 shows the phenotypic group correlations, which broadly mirrored the pattern of $r_{\mathrm{ph}}$ estimates in the full sample. Communication displayed stronger associations with both Conners ADHD subscales (53-60) than RRBI (24-40) and Social (24-34). In the univariate analyses, DZ co-twin means were closer to 0 than MZ co-twin means, indicating $h_{\mathrm{g}}^{2}$ for each subscale (see Table S2). Regression analyses confirmed this; $h_{\mathrm{g}}^{2}$ estimates, which are shown in Table S3, ranged from .69-.73 for the CAST subscales, and from .70-.85 for the Conners ADHD subscales. In the bivariate analyses, all DZ co-twin means on the outcome measure regressed toward 0 to a greater extent than MZ co-twin means, suggesting $h_{2 . x y}$ (see Table S2). Further, bivariate heritability estimates, given in Table S3, indicated that phenotypic group correlations between the CAST and Conners ADHD subscales were almost entirely explained by genetic factors.

From the $h_{\mathrm{g}}^{2}$ and $h_{2 . \mathrm{xy}}$ estimates derived from the DeFries-Fulker analyses, genetic correlations between extreme scores on the CAST and Conners ADHD subscales were calculated. Generally, these were higher than $r_{\mathrm{A}}$ estimates derived in the full sample. The highest estimate was between communication and inattention (.65); communication also displayed considerable genetic overlap with hyperactivity/impulsivity (.60). RRBI displayed moderate genetic overlap with both Conners ADHD subscales (.35.36). Genetic overlap between Social and the two Conners ADHD subscales was weak (.21) to moderate (.45).

\section{Discussion}

We tested the degree of genetic and environmental overlap between specific autistic traits and specific traits of ADHD in the general population, including, for the first time, at the extremes of the general population. The strongest genetic associations, in both the full sample and at the extremes, were between communication difficulties characteristic of ASC and traits of ADHD, with little differentiation in the magnitude of this association across different traits of ADHD. There was also a modest degree of phenotypic and genetic overlap between repetitive routines and interests, and traits of ADHD, and least overlap was observed for autistic social difficulties. As expected, the degree of aetiological overlap between autistic traits and traits of ADHD varied across different types of autistic trait.

In contrast with our expectations, and with the two existing twin studies on this subject $[20,21]$, we did not find that non-social autistic traits showed the strongest association between autistic and ADHD behaviours. It is notable, however, that we did still find, in some instances, moderate genetic overlap between repetitive routines and interests and traits of ADHD, at the extremes and with hyperactivity in the full sample. Hence, our findings do support the notion that traits of ADHD do, at least, show stronger associations with non-social than social autistic traits. The key question is why communication difficulties, specifically, showed the strongest overlap with traits of ADHD.

Item overlap between the CAST Communication subscale and the Conners ADHD subscales represents one possible explanation for why traits of ADHD were so strongly linked with autistic communication difficulties relative to other autistic traits. The CAST Communication subscale is concerned with pragmatic aspects of communication, enquiring specifically about the manner in which the child interacts with other people. The Conners ADHD also asks about these aspects of communication, such as whether the child often struggles to maintain conversations due to their ADHD behaviours. Yet there is considerable evidence that these measures are valid assessments of traits of ASC [25] and ADHD [26], indicating that there can be confidence that these results represent genuine genetic overlap between communication difficulties and traits of ADHD.

From these findings one might predict that individuals with ADHD will be more likely to display communication difficulties similar to those seen in ASC. Indeed, research on the language abilities of individuals with ASC and ADHD are producing somewhat overlapping findings. 
While structural language skills show considerable variability in both these conditions, pragmatic language difficulties appear to feature prominently in both [36, 37]. This pattern might be expected if it is the case that the genes that are associated with communication difficulties characteristic of ASC also link with traits of ADHD.

On a theoretical level, these findings lend additional support to the fractionable autism triad hypothesis [38, 39]. This hypothesis posits that the core features of ASC are each associated with largely different causal influences, and is supported by twin studies [12-17]. These findings extend this hypothesis, by suggesting that the manner in which core autistic traits relate to traits characteristic of ADHD differs across autistic trait domains. Intriguingly, these findings are similar to those reported in a twin study, also conducted on the TEDS sample, that suggested heightened aetiological overlap between communication difficulties and internalising traits [40], and also reflect those of a recent longitudinal twin study of the phenotypic associations between ASC and ADHD [8]. Thus, this study adds to increasing evidence to suggest that the fractionable autism triad hypothesis extends to how autistic traits relate to traits of other conditions.

Findings such as these have the potential to inform molecular genetic research endeavours targeted at identifying genetic variants that are shared across ASC and other conditions. This is clearly a distal goal; most molecular genetic studies have focused on ASC and ADHD as single constructs. For instance, one study reported that genetic variants, specifically calcium-signalling genes, were associated with both ASC and ADHD [41]. Could these variants be associated, in particular, with communicative aspects of ASC? Research taking a symptom-specific approach to ASC is scarce [e.g. 42]. Encouragingly, a recent paper, using data from the ALSPAC sample, tested the association between polygenic scores for ADHD and various neurodevelopmental difficulties, including autistic traits. Of note, the authors reported that while polygenic scores for ADHD did not significantly associate with social cognition difficulties, they were particularly linked with pragmatic language abilities [43], which is a similar pattern to that seen in the present study. Thus, our findings, along with these independent results, suggest that this is a potentially fruitful future direction for molecular genetic research on ASC and ADHD overlap.

These findings need to be regarded in light of the limitations of this study. Owing to the large sample size, requisite for twin model fitting to be adequately performed and for sufficient probands to be identified for extremes analysis, we were unable to conduct in-depth assessments on participants. However, this need not be viewed as a limitation. There is evidence that the heritability of traits of ASC and ADHD is constant across the general population and at the extremes $[5,6]$, and we did find similar, albeit slightly stronger, associations at the extremes of our sample. Furthermore, we were able to avoid referral biases that can be present in clinically ascertained samples, which can inflate co-occurrence rates between conditions. One might question whether these findings from twins generalise to singletons. However, autistic traits and traits of ADHD do not appear to be elevated in twins relative to singletons $[44,45]$. Future studies should also focus on assessments of ASC and ADHD from multiple raters, rather than parents alone. The internal consistency of the three CAST subscales was relatively low, as noted previously [13, 14]. Particular items failing to contribute to the internal consistency sufficiently did not account for this, since removing any items did not result in discernable improvements in internal consistency. The modest internal consistency is, perhaps, due to the relatively small number of items that comprise each of the subscales, or heterogeneity within each subscale. An important future direction, then, is to assess autistic traits in a manner that maximises reliability, although good construct validity for the CAST subscales has been reported previously [13, 14].

This study demonstrated that the degree to which autistic traits and traits of ADHD share genetic and environmental influences with one another varies by autistic trait domain, showing for the first time that this pattern is also apparent amongst individuals in the general population displaying particularly extreme degrees of traits of ASC and ADHD. Autistic communication difficulties, in particular, were associated with similar genetic influences to traits of ADHD, more so than autistic social or non-social behaviours. These findings from a community sample suggest the importance of taking a trait-specific approach to understanding why ASC co-occurs with other conditions, rather than focusing on the ASC phenotype as a whole. These findings may lead to the prediction that communication difficulties characteristic of ASC will present with increased frequency in individuals with ADHD. Indeed, similar communication difficulties, such as pragmatic difficulties, have been reported in both ASC and ADHD [36, 37]; our findings offer a potential explanation with regard to these findings by suggesting that similar causes are associated with both traits of ADHD and communication difficulties characteristic of ASC.

Acknowledgments We gratefully acknowledge all participating families and researchers involved in TEDS for making this study possible. We thank Professor Robert Plomin for this research collaboration. TEDS is funded by a programme grant from the UK Medical Research Council [G0901245; and previously G0500079]. The first author was funded by the UK Medical Research Council and Economic and Social Research Council.

Conflict of interest On behalf of all authors, the corresponding author states that there is no conflict of interest. 


\section{References}

1. American Psychiatric Association (2013) Diagnostic and statistical manual of mental disorders, 5th edn. American Psychiatric Association, Washington

2. Simonoff E, Pickles A, Charman T, Chandler S, Loucas T, Baird G (2008) Psychiatric disorders in children with autism spectrum disorders: prevalence, comorbidity, and associated factors in a population-derived sample. J Am Acad Child Adolesc Psychiatry 47:921-929. doi:10.1097/CHI.0b013e318179964f

3. Yoshida Y, Uchiyama T (2004) The clinical necessity for assessing attention deficit/hyperactivity disorder (AD/HD) symptoms in children with high-functioning pervasive developmental disorder. Eur Child Adolesc Psychiatry 13:307-314. doi:10.1007/ s00787-004-0391-1

4. Reiersen AM, Constantino JN, Volk HE, Todd RD (2007) Autistic traits in a population-based ADHD twin sample. J Child Psychol Psychiatry 48:464-472. doi:10.1111/j.1469-7610.2006.01720.x

5. Ronald A, Hoekstra RA (2011) Autism spectrum disorders and autistic traits: a decade of new twin studies. Am J Med Genet B Neuropsychiatr Genet 156B:255-274. doi:10.1002/ajmg.b.31159

6. Posthuma D, Polderman TJ (2013) What have we learned from recent twin studies about the etiology of neurodevelopmental disorders? Curr Opin Neurol 26:111-121. doi:10.1097/ WCO.0b013e32835f19c3

7. Ronald A, Simonoff E, Kuntsi J, Asherson P, Plomin R (2008) Evidence for overlapping genetic influences on autistic and ADHD behaviours in a community twin sample. J Child Psychol Psychiatry 49:535-542. doi:10.1111/j.1469-7610.2007.01857.x

8. Taylor MJ, Charman T, Robinson EB, Plomin R, Happé F, Asherson P, Ronald A (2013) Developmental associations between traits of autism spectrum disorder and attention deficit hyperactivity disorder: a genetically informative, longitudinal twin study. Psychol Med 43:1735-1746. doi:10.1017/S003329171200253X

9. Lichtenstein P, Carlström E, Råstam M, Gillberg C, Anckarsäter H (2010) The genetics of autism spectrum disorders and related neuropsychiatric disorders in childhood. Am J Psychiatry 167:1357-1363. doi:10.1176/appi.ajp.2010.10020223

10. Lundström S, Chang Z, Kerekes N, Gumpert CH, Råstam M, Gillberg C, Lichtenstein P, Anckarsäter H (2011) Autistic-like traits and their association with mental health problems in two nationwide twin cohorts of children and adults. Psychol Med 41:2423-2433

11. Reiersen AM, Constantino JN, Grimmer M, Martin NG, Todd RD (2008) Evidence for shared genetic influences on self-reported ADHD and autistic symptoms in young adult Australian twins. Twin Res Hum Genet 11:579-585. doi:10.1375/twin.11.6.579

12. Ronald A, Happé F, Plomin R (2005) The genetic relationship between individual differences in social and nonsocial behaviours characteristic of autism. Dev Sci 8:444-458. doi:10.1111/j.1467-7687.2005.00433.x

13. Ronald A, Happé F, Bolton P, Butcher LM, Price TS, Wheelwright S, Baron-Cohen S, Plomin R (2006) Genetic heterogeneity between the three components of the autism spectrum: a twin study. J Am Acad Child Adolesc Psychiatry 45:691-699. doi:10.1097/01.chi.0000215325.9d

14. Ronald A, Happé F, Price TS, Baron-Cohen S, Plomin R (2006) Phenotypic and genetic overlap between autistic traits at the extremes of the general population. J Am Acad Child Adolesc Psyciatry 45:1206-1214. doi:10.1097/01.chi.0000230165.54117.41

15. Robinson EB, Koenen KC, McCormick MC, Munir K, Hallett V, Happé F, Plomin R, Ronald A (2012) A multivariate twin study of autistic traits in 12-year-olds: testing the fractionable autism triad hypothesis. Behav Genet 42:245-255. doi:10.1007/ s10519-011-9500-3
16. Ronald A, Larsson H, Anckarsäter H, Lichtenstein P (2011) A twin study of autism symptoms in Sweden. Mol Psychiatry 16:1039-1047. doi:10.1038/mp.2010.82

17. McLoughlin G, Ronald A, Kuntsi J, Asherson P, Plomin R (2007) Genetic support for the dual nature of attention deficit hyperactivity disorder: substantial genetic overlap between the inattentive and hyperactive-impulsive components. J Abnorm Child Psychol 35:999-1008. doi:10.1007/s10802-007-9149-9

18. Greven CU, Rijsdijk FV, Plomin R (2011) A twin study of ADHD symptoms in early adolescence: hyperactivity-impulsivity and inattentiveness show substantial genetic overlap but also genetic specificity. J Abnorm Child Psychol 39:265-275. doi:10.1007/ s10802-010-9451-9

19. McLoughlin G, Rijsdijk F, Asherson P, Kuntsi J (2011) Parents and teachers make different contributions to a shared perspective on hyperactive-impulsive and inattentive symptoms: a multivariate analysis of parent and teacher ratings on the symptom domains of ADHD. Behav Genet 41:668-679. doi:10.1007/ s10519-011-9473-2

20. Polderman TJ, Hoekstra RA, Vinkhuyzen AA, Sullivan PF, van der Sluis S, Posthuma D (2013) Attentional switching forms a genetic link between attention problems and autistic traits in adults. Psychol Med 43:1985-1996. doi:10.1017/ S0033291712002863

21. Ronald A, Larsson H, Anckarsater H, Lichtenstein P (2014) Symptoms of autism and ADHD: a Swedish twin study examining their overlap. J Abnorm Psychol 123:440-451. doi:10.1037/ a0036088

22. Haworth CM, Davis OS, Plomin R (2013) Twins early development study (TEDS): a genetically sensitive investigation of cognitive and behavioral development from childhood to young adulthood. Twin Res Hum Genet 16:117-125. doi:10.1017/ thg.2012.91

23. Price T, Freeman B, Craig I, Petrill SA, Ebersole L, Plomin R (2000) Infant zygosity can be assigned by parental report questionnaire data. Twin Res 3:129-133. doi:10.1375/136905200320565391

24. Scott FJ, Baron-Cohen S, Bolton P, Brayne C (2002) The CAST (Childhood Asperger Syndrome Test): preliminary development of a UK screen for mainstream primary-school-age children. Autism 6:9-31. doi:10.1177/1362361302006001003

25. Williams J, Scott F, Stott C, Allison C, Bolton P, Baron-Cohen S, Brayne C (2005) The CAST (Childhood Asperger Syndrome Test): test accuracy. Autism 9:45-68. doi:10.1177/ 1362361305049029

26. Conners CK, Sitarenios G, Parker JDA, Epstein JN (1998) The revised Conners' Parent Rating Scale (CPRS-R): factor structure, reliability, and criterion validity. J Abnorm Child Psychology 26:257-268. doi:10.1023/A:1022602400621

27. Neale MC, Boker SM, Xie G, Maes HH (2003) Mx: statistical modeling. Virginia Commonwealth University, Richmond

28. McGue M, Bouchard TJ (1984) Adjustment of twin data for the effects of sex and age. Behav Genet 14:325-343. doi:10.1007/ BF01080045

29. Loehlin JC (1996) The Cholesky approach: a cautionary note. Behav Genet 26:65-69. doi:10.1007/BF02361160

30. Markon KE, Krueger RF (2004) An empirical comparison of information-theoretic selection criteria for multivariate behavior genetic models. Behav Genet 34:593-610

31. Raftery AE (1995) Bayesian model selection in social research. Social Methodol 25:111-163

32. DeFries JC, Fulker DW (1985) Multiple regression analysis of twin data. Behav Genet 15:467-473. doi:10.1007/BF01066239

33. Light JG, DeFries JC (1995) Comorbidity of reading and mathematics disabilities: genetic and environmental etiologies. J Learn Disabil 28:96-106. doi:10.1177/002221949502800204 
34. Purcell S, Eley TC, Dale PS, Oliver B, Petrill SA, Price TS, Saudino KJ, Simonoff E, Stevenson J, Taylor E, Plomin R (2001) Comorbidity between verbal and non-verbal cognitive delay in 2-year-olds: a bivariate twin analysis. Dev Sci 4:195-208

35. Knopik VS, Alarcón M, DeFries JC (1997) Comorbidity of mathematics and reading deficits: evidence for a genetic etiology. Behav Genet 27:447-453

36. Geurts HM, Embrechts M (2008) Language profiles in ASD, SLI, and ADHD. J Autism Dev Disord 38:1931-1943. doi:10.1007/ s10803-008-0587-1

37. Geurts HM, Verté S, Oosterlaan J, Roeyers H, Hartman CA, Mulder EJ, Berckelaer-Onnes IA, Sergeant JA (2004) Can the Children's Communication Checklist differentiate between children with autism, children with ADHD, and normal controls? J Child Psychol Psychiatry 45:1437-1453. doi:10.1111/j.1469-7610.2004.00326.x

38. Happé F, Ronald A, Plomin R (2006) Time to give up on a single explanation for autism. Nat Neurosci 9:1218-1220. doi:10.1038/ $\mathrm{nn} 1770$

39. Happé F, Ronald A (2008) The 'fractionable autism triad': a review of evidence from behavioural, genetic, cognitive and neural research. Neuropsychol Rev 18:287-304. doi:10.1007/ s11065-008-9076-8

40. Hallett V, Ronald A, Rijsdijk F, Happé F (2012) Disentangling the associations between autistic-like and internalizing traits: a community based twin study. J Abnorm Child Psychol 40:815827. doi:10.1007/s10802-011-9596-1

41. Cross-Disorder Group of the Psychiatric Genomics Consortium (2013) Genetic relationship between five psychiatric disorders estimated from genome-wide SNPs. Nat Genet 45:984-994. doi: $10.1038 / n g .2711$

42. Ronald A, Butcher LM, Docherty S, Davis OS, Schalkwyk LC, Craig IW, Plomin R (2010) A genome-wide association study of social and non-social autistic-like traits in the general population using pooled DNA, $500 \mathrm{~K} \mathrm{SNP}$ microarrays and both community and diagnosed autism replication samples. Behav Genet 40:3145. doi:10.1007/s10519-009-9308-6

43. Martin J, Hamshere ML, Stergiakouli E, O’Donovan MC, Thapar A (2014) Genetic risk for attention-deficit/hyperactivity disorder contributes to neurodevelopmental traits in the general population. Biol Psychiatry (epub ahead of print). doi:10.1016/j. biopsych.2014.02.013

44. Curran S, Dworzynski K, Happé F, Ronald A, Allison C, BaronCohen S, Brayne C, Bolton PF (2011) No major effect of twinning on autistic traits. Autism Res 4:377-382. doi:10.1002/aur.207

45. Moilanen I, Linna SL, Ebeling H, Kumpulainen K, Tamminen T, Piha J, Almqvist F (1999) Are twins' behavioural/emotional problems different from singletons? Eur Child Adolesc Psychiatry $8: 62-67$ 